\title{
Small-intestinal hemorrhage caused by treatment with sorafenib for hepatocellular carcinoma and diagnosed by capsule endoscopy
}

Sorafenib, a multikinase inhibitor, is the first-line treatment for unresectable progressive hepatocellular carcinoma (HCC) and has been approved by both the United States Food and Drug Administration (FDA) and the European Medicines Agency (EMEA) [1,2]. Although there are studies reporting its effectiveness, there are also reports about adverse events related to treatment with this drug. Among the noted side effects is gastrointestinal hemorrhage, although only a few studies have reported the frequency of this complication $[3,4]$. This is the first case report of small-intestinal hemorrhage following sorafenib treatment, and includes a description of the finding of hemorrhagic lesions as determined by capsule endoscopy. An 80-year-old woman with hepatitis C liver cirrhosis and being treated at a local hospital underwent ultrasound, which showed a mass lesion in the S8 region ( $40 \mathrm{~mm}$ in diameter). She was referred to our hospital for further investigation and treatment of the mass lesion. Based on the findings of dynamic computed tomography (CT) and ethoxybenzyl magnetic resonance imaging (EOB-MRI), the patient was diagnosed as having HCC. We recommended surgical treatment and transcatheter arterial chemoembolization (TACE) but the patient declined this treatment option. Therefore, sorafenib $400 \mathrm{mg}$ /day was started on day 20 in the hospital. On day 28 , tarry stools were observed, and serum chemistry investigations revealed red blood count $369 \times 10^{4} / \mu \mathrm{L}$, hemoglobin $11.8 \mathrm{~g} / \mathrm{dL}$, and hematocrit $34.1 \%$, which suggested normocytic hypochromic anemia. On day 30, esophagogastroduodenoscopy revealed no hemorrhagic esophageal varices with $\operatorname{LiF} 1 \mathrm{CbRC}(-)$ ( $\bullet$ Fig.1). On day 35, colonoscopy revealed dark blood throughout the colon but no evident hemorrhagic lesions ( Fig. 2). On day 36, the small intestine was examined with a capsule endoscope. Oozing of blood from several hemorrhagic lesions was observed. This was diagnosed as small-

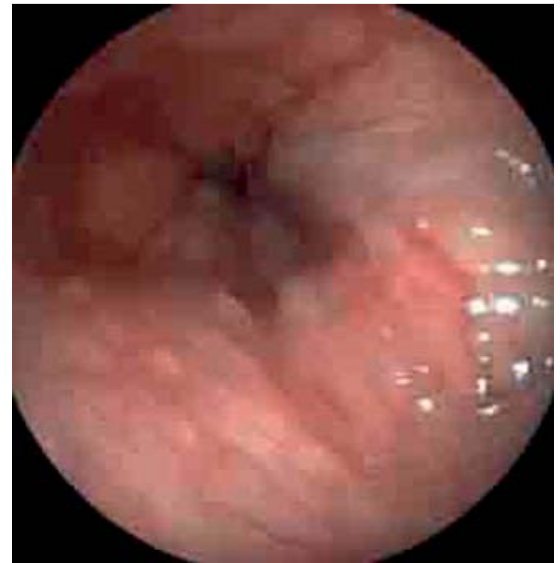

Fig. 1 Esophagogastroduodenoscopy in an 80-year-old woman with hepatocellular carcinoma and tarry stools showing no hemorrhagic varices (LiF1CbRC(-)) in the esophagus.

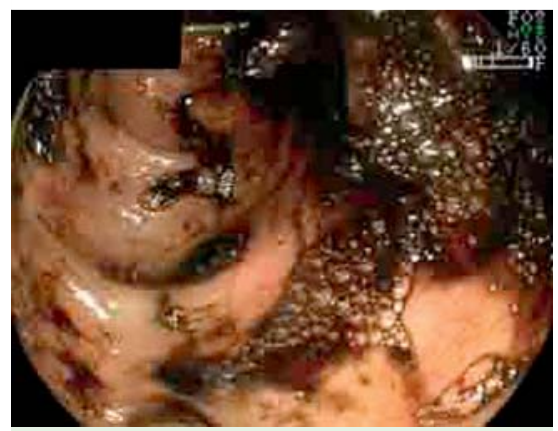

Fig. 2 Colonoscopy showed no active hemorrhagic lesions.

intestinal hemorrhage ( $\bullet$ Fig. 3 ) as a side effect of sorafenib therapy. The symptoms quickly improved on cessation of the treatment. Capsule endoscopy on day 90 showed scarlike lesions in the distal small intestine, indicating significant improvement ( Fig.4).

To our knowledge, this is the first report of hemorrhage in the small intestine caused by sorafenib treatment and diagnosed by capsule endoscopy.

Endoscopy_UCTN_Code_CCL_1AB_2AZ_3AD

Competing interests: None
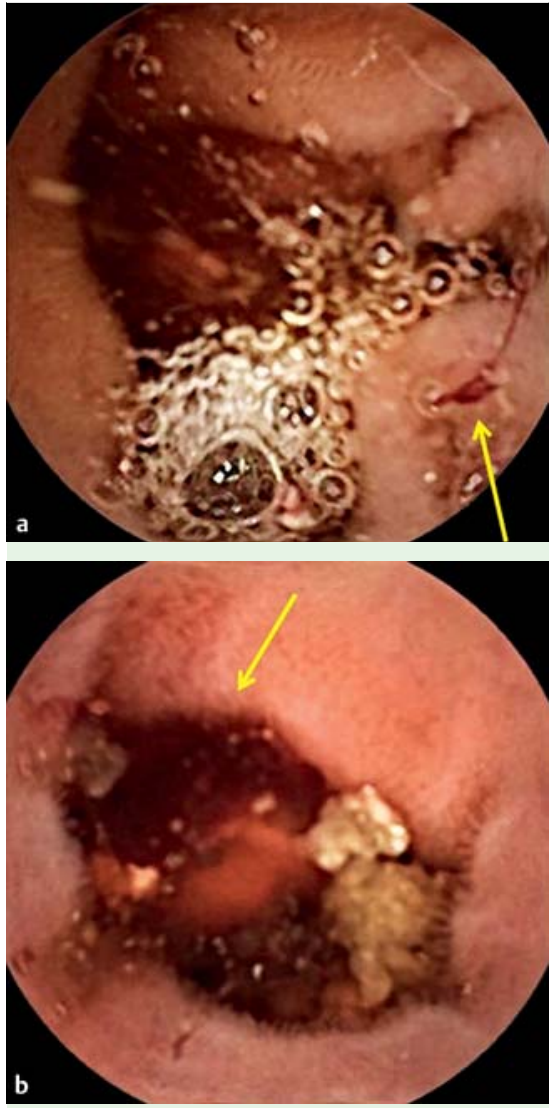

Fig. 3 First report of hemorrhagic lesions in the small intestine detected by capsule endoscopy (arrows). a Proximal small intestine. b Distal small intestine.

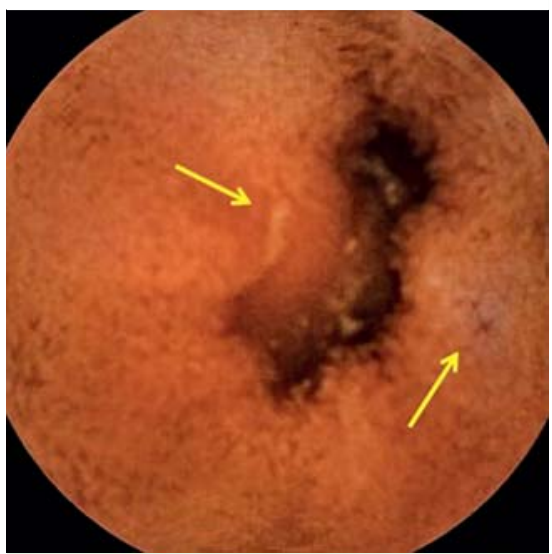

Fig. 4 Second capsule endoscopy of the small intestine showing improvement (arrows). 
Y. Takahashi' ${ }^{1,2}$, S. Fukunishi ${ }^{2}$, T. Nishikawa $^{1,2}$, S. Nouda ${ }^{2}$, Y. Sasaki ${ }^{1}$, M. Sanomura' , E. Umegaki², K. Higuchi ${ }^{2}$

${ }^{1}$ Department of Gastroenterology, Hokusetsu General Hospital, Osaka, Japan

2 2nd Department of Internal Medicine, Osaka Medical College, Osaka, Japan

\section{References}

1 Wilhelm SM, Carter C, Tang $l$ et al. BAY 439006 exhibits broad spectrum oral antitumor activity and targets the RAM/MEK/ ERK pathway and receptor tyrosine kinase involved in tumor progression and angiogenesis. Cancer Res 2004; 64: 7099-7109

2 Chang YS, Adnane JZ, Trail PA et al. Sorafenib (BAY 43-9006) inhibits tumor growth and vascularization and induces tumor apoptosis and hypoxia in RCC xenograft models. Cancer Chemother Pharmacol 2007; 59: $561-574$

3 Llovet JM, Ricci S, Mazzeferro V et al. Sorafenib in advanced hepatocellular carcinoma. N Engl J Med 2008; 359: 378 - 390

4 Cheng AL, Kang YK, Chen Z et al. Efficacy and safety of sorafenib in patients in the Asia-Pacific region with advanced hepatocellular carcinoma: a phase randomized, doubleblind, placebo-controlled trial. Lancet Oncol 2009; 10: $25-34$
Bibliography

Dol http://dx.doi.org/

10.1055/s-0032-1326641

Endoscopy 2013; 45: E179-E180

(c) Georg Thieme Verlag KG

Stuttgart · New York

ISSN 0013-726X

\section{Corresponding author}

\section{S. Fukunishi}

2-7 Daigaku-machi

Takatsuki

Osaka 569-8686

Japan

Fax: +81-726-846532

in2104@poh.osaka-med.ac.jp 